\title{
Anreize für die Qualitätssicherung Umsetzung von Pay for Performance (P4P) im Gesundheitswesen
}

Dr. med. Christof Veit ist Experte auf dem Gebiet der Qualitätssicherung im Gesundheitswesen. Im Mai 2007 wurde er zum Geschäftsführer der Bundesgeschäftsstelle Qualitätssicherung gGmbH berufen, dem heutigen BQS Institut für Qualität und Patientensicherheit. Im letzten Jahr schloss sein Institut $u$. a. einen ersten Sachstandsbericht über die Umsetzung von Pay for Performance (P4P) im Gesundheitswesen ab. Das Gutachten wurde 2010 vom Bundesministerium für Gesundheit in Auftrag gegeben. Im Vordergrund stand die Frage, ob es wissenschaftliche Belege gibt, dass die Qualität in der Gesundheitsversorgung durch finanzielle Anreize verbessert werden kann.

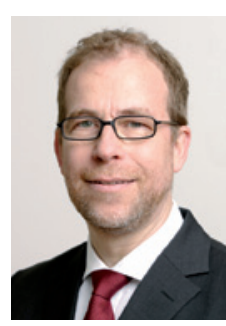

Dr. Christof Veit

\section{Herr Dr. Veit, zu welchem Ergebnis kommt Ihr Gutachten? Ist die Wirksamkeit von P4P erwiesen?}

Dr. Christof Veit: Das Gutachten zeigt, dass eine leistungsorientierte Vergütung grundsätzlich möglich ist, dass man hierbei aber sorgfältig vorgehen muss. Die primäre Motivation der Ärzte und Pfleger lässt sich nicht ersetzen, doch ist es möglich, gute Qualität besser zu honorieren und das System für diejenigen, die sich Qualitätsstandards verweigern, unattraktiv zu machen. Dass finanzielle Anreize mächtig und wirksam sind, zeigen Erfahrungen aus Modellprojekten, die wir in dem Gutachten zusammengetragen haben. Ein zweifelsfreier Nachweis über wissenschaftliche Evidenz der unterschiedlichen P4P-Modelle in Gänze steht allerdings noch aus. Die Studien sind methodisch und sachlich sehr anspruchsvoll.

\section{Was genau verstehen Sie unter} P4P? Gibt es unterschiedliche Definitionen?

Veit: P4P steht für eine Anpassung der Leistungsvergütung an die erbrachte Qualität, genauer an die Ergebnisqualität der Versorgung. Das ist neu. Es geht darum, ob die Patienten nach der Behandlung den Gesundheitszustand erlangt haben, den sie tatsächlich erreichen konnten, wenn man beispielsweise ihr Alter und Vorerkrankungen oder ihre Compliance berücksichtigt. Eine gute Versorgung soll besser bezahlt werden als eine schlechte. Dies kann auf 3 Wegen gesche- hen: 1) Die Vergütungstöpfe werden aufgestockt, damit steht zusätzliches Geld für die Honorierung zur Verfügung. 2) Es erfolgt eine Umverteilung von „schlecht“ nach „gut“, was für einzelne Häuser und Praxen mit finanziellen Einbußen verbunden ist. 3) Das Sharing-Modell basiert darauf, dass eine hohe Versorgungsqualität effizient ist und Folgekosten einspart. Diese „Gewinne“ können dann zwischen Leistungserbringern und Kostenträgern aufgeteilt werden.

? Wie lässt sich die Qualität messen? Veit: Versorgungsqualität zu messen, ist ein schwieriges Unterfangen, da Behandlungsergebnisse verschiedenen Einflüssen unterliegen. Dennoch stehen wir nicht am Anfang. Es gibt bereits zahlreiche Indikatoren, die genutzt werden können. Der Gesetzgeber schreibt nach § 137 SGB V die externe Qualitätssicherung für Krankenhäuser vor. Mittlerweile liegen 430 Indikatoren zu 30 Leistungsbereichen vor. Ein Indikator ist mehr als eine Kennziffer, da die Qualitätssicherer zusätzlich auf Basis der vorhandenen Studienlage prüfen, ob der Wert innerhalb eines Referenzbereichs liegt oder schlechter ist. Die Ergebnisse werden den Krankenhäusern zurückgespiegelt. Weitere Daten resultieren aus den Abrechnungsverfahren mit den Krankenkassen nach $\S$ 21 KHEntG. Sie enthalten Diagnosen nach ICD und Prozeduren nach OPS sowie Hinweise auf Eingriffe wegen Komplikationen. Portale wie Qualitätskliniken.de werten auch diese Daten sowie Daten zur Patientensicherheit und Zufriedenheit unter Qualitätsaspekten aus, um Krankenhäuser umfassender vergleichen $\mathrm{zu}$ können. Ferner gibt es die Hygiene- und Infektionsstatistiken.

In welchen Fällen bewähren sich leistungs- oder erfolgsorientierte Vergütungsanreize?

Veit: Wenn neue medizinische Standards bekannt sind und die Leitlinien entsprechend aktualisiert wurden, lässt sich in Benchmarking-Projekten prüfen, welche Krankenhäuser und Praxen diese bereits berücksichtigen. Diejenigen, die sich schneller umstellen, sollen auch besser bezahlt werden. Andererseits sollten Krankenhäuser, die beispielsweise zu wenig Prophylaxe betreiben oder bei der Physiotherapie sparen, eine geringere Finanzierung erhalten. Über P4P lässt sich auch zusätzlicher Versorgungsaufwand abbilden, sofern erwiesen ist, dass dadurch tatsächlich bessere Ergebnisse erzielt werden. Dies kann bei Leistungen für die Patientensicherheit oder die Frührehabilitation der Fall sein. Bei selektiven Versorgungsverträgen sind qualitätsbezogene Vergütungsstrukturen bereits üblich. Die Vertragspartner greifen entweder auf vorhandene Indikatoren zurück oder entwickeln eigene, die stärker auf den Vertragsinhalt zugeschnitten sind. In unserem Gutachten sind einige beschrieben, zum Beispiel enthalten die IVVerträge zu Kopfschmerzen und Rückenschmerzen P4P-Elemente, die als Ergebnisparameter die Wiedererlangung der $\mathrm{Ar}$ beitsfähigkeit messen.

\section{Wo sehen Sie Grenzen finanzieller} Anreize zur Qualitätssicherung?

Veit: Die primäre Motivation der helfenden Berufe lässt sich durch Geld nicht herstellen. Mit den finanziellen Anreizen für höchste Qualität können die Kliniken aber dafür Sorge tragen, dass die Rahmenbedingungen der Arbeit so gestaltet sind, dass die Ärztinnen und Schwestern, Ärzte und Pfleger gute Qualität in der Versorgung erbringen können, sodass sie motiviert bleiben. Wenn wir die Qualität bewerten, dürfen wir in der Bewertung nur das zugrunde legen, was mit den Instrumenten tatsächlich zuverlässig gemessen und ggf. einrichtungsübergreifend verglichen werden kann. Hier müssen wir noch einige Entwicklungsarbeit in gute Qualitätsmessinstrumente stecken.

Qualitätssicherung strebt an, allen Patienten eine gute und sichere Behandlung zu ermöglichen. Ist dies überhaupt realistisch? 
Veit: Eine $100 \%$ ige Sicherheit ist nicht realistisch, menschliche Irrtümer wird es immer geben können. Die Geschichte der Medizin zeigt aber, dass Verfahren und Techniken immer sicherer geworden sind. Und wir arbeiten daran, uns kontinuierlich weiter zu verbessern.

\section{? Wie erkennen Patienten schlechte Versorgungsqualität?}

Veit: Patienten erleben Komplikationen nach einer Operation oder konservativen Therapie oft schmerzhaft am eigenen Körper, zum Beispiel bei Wundinfektionen. Im Vorfeld die Güte der Versorgung zu erkennen ist schwierig. Patienten sollten darauf vertrauen können, dass alle Krankenhäuser eine gleich gute Behandlungsqualität auf ihrem Gebiet bieten. Wir wissen aber, dass es Unterschiede gibt. Das zeigt u.a. die externe Qualitäts- sicherung. Die Informationen sind in den Qualitätsberichten, die öffentlich gemacht werden müssen, prinzipiell enthalten. Sie sind aber schwer verständlich und werden weder von den zuweisenden Ärzten noch von den Patienten bisher ausführlich genutzt. Bei der Informationstransparenz besteht noch Verbesserungsbedarf. Internetportale wie www. qualitaetskliniken.de und www.weisseliste.de sind ein wichtiger Schritt in diese Richtung.

\section{? Was zeichnet Krankenhäuser mit einer hohen Qualität aus? Was machen sie anders als andere Häuser?}

Veit: Zu dieser Frage laufen gerade spannende Forschungsprojekte. Es zeichnet sich ab, dass Häuser, die in den Benchmarks gut abschneiden, selbstkritischer mit ihren Ergebnissen umgehen und fort- laufend nach weiteren Verbesserungspotenzialen suchen. Außerdem gilt Qualitätssicherung dort als eine Gemeinschaftsaufgabe, um die man sich im Team kümmert. Bei Fehlern geht es nicht um eine individuelle Schuldzuweisung, sondern um gemeinsames Lernen und den Erfahrungsaustauch, sodass ähnliche Fehler künftig vermieden werden können. Hohe Qualität spiegelt sich in der Krankenhauskultur und Organisation wider. Die Häuser schaffen Rahmenbedingungen, die es ermöglichen, dass die Beschäftigten ihre besten Qualitäten zum Einsatz bringen.

Herr Dr. Veit, vielen Dank für das Gespräch!

Das Interview führte Dr. Adelheid Weßling, Düsseldorf. 